\title{
A NUMERICAL ANALYSIS OF CRACK PROPAGATION IN MICROCRACKING CERAMIC AND CERAMIC COMPOSITES
}

\author{
S.B. BINER
}

Ames Laboratory

Iowa State University

Ames, Iowa 50011

\begin{abstract}
:
In this study a set of numerical analyses of crack growth was performed to elucidate the mechanism of microcracking on the observed fracture behavior of ceramics and ceramic composites. The random nucleation, orientation and size effects of discrete microcracks and resulting interactions are fully accounted for in a hybrid finite element model. The results indicate that the energy expenditure due the microcrack nucleation seems not to contribute significantly to the resistance to crack growth. The main controlling parameter appears to be elastic interaction of the microcracks with the main crack in the absence of a reinforcing phase; therefore, the microcrack density plays an important role. In the case of the composites, the interaction of the main crack with the stress fields of the reinforcing phase, rather than interaction of microcracks, is the controlling parameter for the resistance to the crack growth even in the presence of a large population of microcracks. It will be also shown that the crack branching and crack kinking can readily develop as a result of microcracking.
\end{abstract}

\section{INTRODUCTION :}

At least two effects of formation of a microcrack zone ahead of a main crack tip have been well identified with cortrolled experiments and with consistency of among the data generated(1-5). First, the microcracking zone can lower the applied stress intensity factor $\mathrm{K}_{\mathrm{app}}$ at the near-tip of main crack (e.g. $K_{\text {tip }}<K_{\text {app }}$ ) and this shielding effect provides a mechanism for increase in toughness. Secondly, the microcracks can also amplify the local stress intensity (e.g $\mathrm{K}_{\text {tip }}>\mathrm{K}_{\text {app }}$ ) causing very large crack extension or unstable crack growth. Several theoretical studies on microcracking behavior have resulted often in controversy, with findings either with experimental

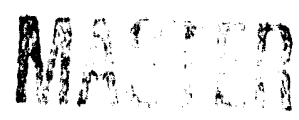


observations or with each other. The interaction of microcracks with a main crack has been modelled using two approaches, namely:

i)- Discrete Microcracking Models, in which limited number of cracks were placed at predetermined locations in the near-tip singular stress field of a main crack. The interaction of stress fields of microcracks with each other and with main crack is solved either by enforcing traction free macrocrack surface conditions (6) or with a superposition and self-consistency schemes to determine the average traction on the individual cracks(7-9). The latter technique yielded analytical solutions for two as well as three dimensional closely-spaced array of microcracks. The interactions of two dimensional microcracks with a main crack were also studied using point source representation and a self-consistency scheme to determine the microcrack orientations which will favor the toughening behavior(10). Other numerical solutions for crack interactions can also found in the literature (11-13).

ii) Continuum Microcracking Models, which are based on the development of a constitutive relationship to describe the stress-strain behavior of solids containing a set of randomly distributed finite microcracks (of penny shape). The resulting reduction in the elastic moduli for the microcrack zone ahead of the main crack has been calculated using self-consistency $(14,15)$ and perturbation methods inclurding the effects of residual stresses(16). The constitutive laws relating the magnitude of stresses to microcrack density including the role of microstructural features have been also developed $(17,18)$. The common feature in these models is the presence of a saturation microcrack density above which further microcrack formation does not occur with increasing stress levels.

It has been shown that the microcracks closest to the main crack produce a dominant effect on the $\mathrm{K}_{\text {rip }}$ resulting in either shielding or amplification and this is very sensitive to the individual microcrack position, orientation and size. Since the random microcrack locations did not produce any statistically stable effect of shielding at the main crack tip, therefore, other mechanisms have been suggested for the toughening behavior resulting from the microcracking. Such as energy expenditure on the nucleation of the microcracks rather than elastic interactions(9) and the 
formation of secondary loading modes (i.e. mode II and mode III stress intensities under mode I loading) which can cause the formation of kinked cracks $(5,9)$. Therefore, it is argued that the modelling of microcrack zone by an elastic material of reduced stiffness would not resolve these local events with enough sensitivity. One other result of interest is that microcracks in the wake region (behind the main crack tip) were found to have little or no effect on the toughening mechanism by shielding(9). However, that the opposite was clearly substantiated in experimental studies of Knehens and Steinbrech(3) where the removal of the formed wake region, by machining, reduced the subsequent crack-growth resistance of the material.

As discussed in (18) a continuum description of the microcracking may provide an effective technique for reasonable estimation the role of microcrack densities on shielding. Also, microcrack-induced anisotropies and residual stress effects can be studied without extensive computation. In discrete microcracking models two basic assumptions are usually made in order to reduce the complexities to tractable levels: the microcracks were chosen to be two dimensional , and prenucleated or nucleation of microcracks (if included in the solution) takes place at predetermined positions. These models require extensive calculations, in particular when a large set of microcracks is introduced. However, it is evident from the above discussion that they bring useful insight to the micromechanism. A good urderstanding of such a micromechanism could be most valuable in the design of these types of brittle materials and composite systems through intelligent manipulation of the microstructure.

In order to address the above conflicting issues, for this study a set of numerical analyses of crack growth was performed to elucidate the mechanism of microcracking on the observed fracture behavior of brittle solids and composites. The random nucleation, orientation and size effects of discrete microcracks and resulting interactions are fully accounted for in a hybrid finite element model.

\section{DETAILS OF THE FEM ANALYSES:}

When the crack configuration is complicated or in the presence of multiple cracks, even by using 
higher order special crack tip singularity elements, to achieve accurate solutions with the conventional finite element methods is very difficult. To overcome overwhelming difficulties, several element formulations have been sought in which the full crack field is represented and not just the crack tip singularity. Thus one element can then be used to model the crack affected zone in place of a refined mesh at the crack tip or special crack tip elements. Since such an element truly represents the complete crack field it should be possible to specify the crack to lie anywhere within the element boundary. Therefore crack extensions and nucleation of new cracks can be modelled without the generation of a new finite element mesh. In the development of such crack elements two approaches have been taken. In the analytical approach the element stiffness matrix is formulated using normal energy theorems, but the stress and displacement fields are expressed in terms of well known crack tip singularity fields(mode I and mode II) which in turn are related to the element nodal displacements(19-21). While this formulation satisfies the internal equilibrium and compatibility conditions, the boundary displacement continuity requirement is often violated. Therefore convergence can not be guaranteed. However, the conditions for nonconvergence can be removed by enforcing boundary conformity of displacements using the hybrid technique(22.). It appears that the hybrid element technique theoretically has the all the advantages of the analytical element approach with the added advantages of being fully conforming and having reduced integration requirements.

In the present study two types of crack elements, one for the microcracks and the other for the main crack (as schematically shown in Fig.1) were employed and the details are given in (23). The effects of microcracking ahead of a growing main crack in plane-strain were analyzed as a boundary value problem for a circular region surrounding the crack tip. The outer boundary of this region is far enough away from the crack tip so that the elastic stress field on the boundary remains virtually unaffected by the microcracking. The FEM mesh used in the analyses is shown in Fig.2. The rectangular mesh at the bottom of this figure is surrounded by the mesh in the center which, in tum, is surrounded by the mesh at the top. The dark areas in the bottom rectangular sector indicate the locations and distribution of the reinforcements in the simulated composite microstructures. The initial main crack tip was located at 10 th element from the left in the bottom rectangular sector. Symmetry conditions were enforced through appropriate 
boundary conditions and so only the semicircle region shown in Fig.2 was used in the calculations. The main crack was traction free, and applied displacements to the most outer boundary were characterize $d$ by $K_{I}$ stress intensity factor. The elements shown in the bottom rectangular sector were super-elements consisting of four sub-elements with four nodes in order to be compatible with the crack elements (since linear variation of the displacements along the element edges was assumed in the construction of the crack element stiffness matrices). Therefore, in these super-elements and also in crack elements (containing microcracks) the internal degrees of freedom associated with the internal nodes were eliminated by static condensation before the assembly of the global stiffness matrix. Other regions of the mesh were composed of regular four node elements.

The microcrack zone only was developed in the most inner rectangular region. The condition for microcrack nucleation within the each element is based on the attainment of a critical normal stress at a randomly pre-determined crack plane for that element. This critical value of fracture stress is also assumed to have a normal distribution

$$
f(x)=\frac{1}{\sqrt{2 \pi} s_{n}} \exp \left(-\frac{1}{2}\left(\frac{x-\sigma_{f}}{s_{n}}\right)^{2}\right)
$$

where $s_{\mathrm{n}}$ is the standard deviation, for which a constant value of 0.5 was taken. The $\sigma_{\mathrm{f}}$ is the mean value of the fracture stress and the selection of its values was based on the relationship

$$
K_{\text {IC }}=\sigma_{f} \sqrt{2 \pi x_{0}}
$$

where $x_{0}$ is a length scale proportional to the microcrack size ( 3 to 5 times the microcrack size) and $\mathrm{K}_{\mathrm{IC}}$ is the intrinsic fracture toughness of the matrix. The distribution of the fracture strength for possible nucleation sites is shown in Fig. 3 for two different microcrack sizes. For each possible nucleation site, these fracture strength values were randomly distributed together with the orientation of the microcracks through a random number generating routine. With the attainment of the microcrack nucleation criterion, the standard stiffness matrix of the super- 
elements was transformed to the crack element stiffness matrix and the finite element equations were solved again. The microcrack size was taken as 0.6 times the width of the elements. To simulate the role of microcrack size effect, the analyses were carried out using a slightly coarser mesh than the one shown in Fig.2.

The condition required for the main crack to propagate was the attainment of a intrinsic fracture toughness value $\mathrm{K}_{\mathrm{IC}}$ at the crack tip. With the satisfaction of this criterion the crack was allowed to propagate one element by nodal release technique (boundary conditions for the nodes to be released at the crack tip changed from normal displacement constraint to traction free condition). The finite element equations were then re-solved for the new condition. If the crack extension criterion was still fulfilled the propagation steps were repeated until the stress intensity factor at the main crack tip $K_{\text {ip }}$ fell below the $K_{\mathrm{IC}}$ value. At this stage the outer displacements were increased for a new value of $K_{2 p p}$; overshooting of the crack tip stress intensity factor was always prevented by using very small increments.

For each increment the values of applied stress intensity factor $\mathrm{K}_{\mathrm{app}}$ were also calculated from the most outer ring of elements using

$$
J \cdot=\int\left(U d y-T_{i} \frac{\partial u_{i}}{\partial x} d s\right)
$$

where $U$ is the strain energy density, $T_{i}$ is the traction vector, $u_{i}$ is the displacement vector and $\mathrm{ds}$ is the arc length along the integration contour $\Gamma$. These calculated $\mathrm{K}_{\text {app }}$ values from J-integral, together with stress intensity factor values $K_{\text {tip }}$ evaluated for the main crack tip and the intrinsic fracture toughness $K_{\mathrm{IC}}$ were used in the construction of shielding/amplification diagrams and crack growth resistance curves (R-curve) for advancing cracks. 


\section{RESULTS and DISCUSSION:}

The effects of continuous nucleation on the crack growth behavior in an unreinforced matrix material were investigated for two microcrack sizes. In the first set of calculations the fracture strength for the nucleation of the microcracks was scaled with the microcrack sizes for a constant $\mathrm{K}_{\mathrm{IC}}$ value using Eq.2. The resulting distribution of the fracture strength for the possible nucleation sites was given earlier in Fig.3. The evolution of the damage and development in the microcrack zones are shown in Fig. 4 for different crack lengths. In this figure, the first crack lengths correspond to the condition where the initial main cracks were ready to propagate (i.e. $K_{\text {tip }} \geq K_{I C}$ ). As can be seen from the figure the microcrack zone widths did not change much with crack propagation, in agreement with the experimental observations(24). In the large microcrack case, although the number of nucleation sites was much smaller a larger amount of microcracking was observed due to a much lower the fracture strength for the nucleation (Fig.3). With the growth of the main crack, further nucleation of the microcracks took place mainly in regions near to the main crack. In both cases, at final crack lengths, pockets of undamaged regions within the microcrack zone can also been seen. The variations in the $\mathrm{K}_{\mathrm{app}} / \mathrm{K}_{\text {tip }}$ values with increasing crack length are given in Fig.5. For the small microcrack case, there was not any significant amount of shielding behavior and $\mathrm{K}_{\text {app }} / \mathrm{K}_{\text {tip }}$ values fluctuated around unity for the crack extension up to one quarter of the total crack growth. Whereas in the case of large microcracks the response was more immediate and this combined with larger scale of shielding behavior. The R-curve behaviors $\left(\mathrm{K}_{\mathrm{app}}\right.$ normalized with the intrinsic fracture toughness $\left.\mathrm{K}_{\mathrm{IC}}\right)$ resulting from these simulations are shown in Fig.6. The flat regions in these curves represent the condition where $\mathrm{K}_{\text {tip }}$ stress intensity factor was either equal or greater than the $\mathrm{K}_{\mathrm{IC}}$; and increases cromespond to the condition where $K_{\text {rip }}$ was smaller than the $K_{I C}$, hence the $K_{a p p}$ had to be increased to maintain the crack propagation. As a result of the observed shielding behavior seen in Fig.5, a much larger increase in the R-curve behavior for the case of large microcracks can be seen in Fig.6.

Since for randomly located microcracks did not produce any statistically stable effect of shielding at the macroscopic crack tip, the energy expenditure on the nucleation of the microcracks rather than their elastic interactions was suggested as a possible mechanism for shielding(9). To 
elucidate this behavior the simulation for the large microcrack case was repeated for a microcrack zone containing all the nucleated microcracks (at the same locations with the same orientations) during the previous growth history as pre-nucleated microcracks. The resulting R-curve behavior is compared with the continuous nucleation case in Fig.7. Although the values obtained for the pre-nucleated case are slightly lower than for the continuous nucleation case, as can be seen the difference is not significantly great. Additionally, at the same crack tip locations $K_{\text {app }}$ values fell below the $\mathrm{K}_{\mathrm{IC}}$ values, therefore the evolution of the R-curve with crack extension remained the same.

The role of reinforcement on the crack growth behavior of microcracking composites was investigated for the reinforcement distribution shown in the rectangular sector at the bottom of Fig.2. The volume fraction of the reinforcement was about 19\%; however, since the analyses were carried out in two dimensions, they were continuous in the thickness direction. The reinforcements were also assumed to fail and generate microcracks. Their fracture strength for the microcrack nucleation was assumed to be the same as that of the matrix $\left(\sigma_{\mathrm{f}}=1.995\right)$ with similar strength distribution. It was further assumed that the reinforcing phases have the same intrinsic fracture toughness $\mathrm{K}_{\mathrm{IC}}$ as the matrix; therefore, the same crack propagation criterion was applied when the main crack was crossing the reinforcements. Two simulations were carried out with Young's modulus ratio of reinforcing phase to matrix $\left(E_{d} / E_{m}\right)$ of 2.0 and 0.5 . The evolution of the damage and microcrack zone developments in the composites were compared with the matrix damage forration in Fig.8. In this figure, first crack length corresponds to the condition where initial main cracks were ready to propagate (i.e. $\mathrm{K}_{\mathrm{tip}} \geq \mathrm{K}_{\mathrm{IC}}$ ). For the composite having higher modulus ratio the location of the column of microcrack formation ahead of the main crack tip corresponds to the location of the nearest reinforcement. This behavior is associated with the development of higher stresses in the reinforcements in this composite system. On the other hand the initial damage in the composite with low modulus ratio was similar to the one in the matrix alone. At final crack lengths, although zone widths appears to be similar in both composites, the number of nucleated microcracks is much larger for the low modulus case. The variations in the $\mathrm{K}_{\text {app }} / \mathrm{K}_{\text {tip }}$ values and $\mathrm{R}$-curves were compared with the data obtained for the matrix in Figs. 9 and 10 respectively. At the very initial stages of the crack growth the shielding/amplification events 
in both composites and in the matrix were similar. However, as soon as the main crack encountered the first reinforcement, shielding/amplification characteristics deviated from each other considerably. In the case of high modulus ratio there was a large amplification in the $\mathrm{K}_{\mathrm{app}} / \mathrm{K}_{\text {tip }}$ ratio, whereas in the case of low modulus ratio there was a large shielding. As can be seen from the figure, this behavior was repeated periodically each time the main crack crossed the reinforcing phase. This initial shielding was so large for the low modulus ratio that $K_{\text {app }}$ had to be increased considerably as can be seen from the R-curve. The large scale microcracking with later crack advances in this composite, therefore, is associated with this large elevation of the $\mathrm{K}_{\mathrm{app}}$. Although a large scale microcracking occurred for this case, the increases in the R-curve correspond to the locations where the main crack was crossing the reinforcements. Similarly, in the composite with high modulus ratio the elevations in the R-curve took place just prior to the main crack encounters with the reinforcements.

The distribution of the axial stress ahead of the main cracks is shown for the matrix and for the composite cases in Fig.11. At some microcracks much higher elevation of the stress than at the main crack tip, and formation of the axial compressive stress at some microcracks due the orientation can be clearly seen. In the case of composites besides stress irregularities resulting from microcracking it also important to note the stress distribution in the reinforcing phase. The lower modulus ratio resulted in much lower axial stress in the reinforcements than in the surrounding matrix and the opposite occured for the high modulus case.

From the results obtained it appears that in the absence of a reinforcing phase, the observed $\mathrm{R}$ curve behavior is primarily associated with the elastic interactions of the microcracks with the main crack. The magnitude of the resistance to crack growth increases with increasing microcrack density (Figs.5 and 6). The energy expenditure due to the microcrack nucleation seems not to contribute significantly to toughening behavior (Fig.7) as suggested earlier (5,9). In the case of the presence of reinforcements with rigid interfaces, the resistance to crack growth is due to the interactions of the main crack with stress fields of the reinforcements rather than with the microcracks even in the presence of very large microcrack densities (Figs. 8,9 and 10). The observed much higher stress intensity factors (mode-I and mode-II) at some microcrarks 
(depending on the orientation and location) than at the main crack tip indicate that experimentally observed crack branching and crack kinking $(5,25)$ can readily develop as a result of microcracking. These effects could be significant contributing factors to the toughening besides shielding arising from the elastic interactions of the microcracks.

\section{CONCLUSIONS :}

In this study the crack growth behavior in brittle microcracking solids and composites were numerically investigated. The results indicate that:

1. The energy expanditure due the microcrack nucleation seems not to contribute significantly to the resistance to crack growth. The main controlling parameter appears to be elastic interaction of the microcracks with the main crack in the absence of any reinforcement; therefore, the microcrack density plays an important role.

2. In the case of the composites, the interaction of the main crack with the stress fields of the reinforcing phase, rather than interaction of microcracks, is the controlling parameter for the resistance to the crack growth even in the presence of a large population of microcracks.

\section{ACKNOWLEDGEMENTS :}

This work was performed for the United States Department of Energy by Iowa State University under contract No. W-7405-Eng-82. This research supported by the Director of Energy Research, Office of Basic Energy Sciences.

\section{REFERENCES :}

1. H. Hubner and W. Jillek, J. Mater. Sci. 12, 117, (1977)

2. R.W. Rice, S.W. Freiman and P.E. Becker, J. Amer. Ceram. Soc. 64, 345, (1981)

3. R. Knehens and R. Steinbreck, J. Mater. Sci. Lett. 1, 327, (1982)

4. J.G. Zwissler and M.A. Adams "Fracture Mechanics of Cellular Glass" in Fracture Mechanics of Ceramics (Eds. R.C. Bradt, A.G. Evans, D.P.H. Hasselman and F.F. Lang) 6, 211, (1983) 
(Plenum Press New York)

5. L.X. Han, R. Warren and S. Suresh, Acta Metall. Mater. 40, 259, (1992)

6. R.G. Hoagland and J.D. Embury, J. Amer. Ceram. Soc. 63, 404, (1980)

7. M. Kachanov and E.L.E Montagut, Eng. Fract. Mech. 25, 625, (1986)

8. M. Kachanov and J.P. Laures, Int. J. Fract. 41, 289, (1989)

9. M. Kachanov, E.L.E Montagut and J.P. Lures, Mech. Mater. 10, 59, (1990)

10. L.R.F Rose, J. Amer. Ceram. Soc. 69, 212, (1986)

11. A. Chudnovsky and S. Wu, Int. J. Fract. 42, 43, (1990)

12. A. Rubinstein, Int. J. Fract. 27, 113, (1985)

13. A. Rubinstein, J. Appl. Mech. Trans. ASME 53, 505, (1986)

14. B. Budiansky and R.J. O' Connell, Int. J. Solids and Struct. 12,81, (1975)

15. N. Laws and J.R. Brockenbrough, Int. J. Solids and Struct. 23, 1247, (1987)

16. J.W. Hutchinson, Acta Metall Mater. 35, 1605, (1987)

17. A.G. Evans and K.T. Faber, J. Amer. Ceram. Soc. 67, 255, (1984)

18. P.G. Charambides and R.M. McMeeking, Mech. Mater. 6, 71, (1987)

19. A.K. Rao, I. Raju and A.K. Murty, Int. J. Num. Meth. Engng. 3, 389, (1971)

20. E. Byskov, Int. J. Fract. Mech. 6, 159, (1970)

21. A.J. Fawkes, D.R.J. Owen and A.R. Luxmore, Eng. Fract. Mech. 11, 143, (1979)

22. P. Tong, T.H.H. Pian and S.J. Lasry, Int. J. Num. Meth. Engng. 7, 297, (1973)

23. S.B. Biner (to be published).

24. D.M. Marshall, Presentation at the Winter Study Group, University of California, Santa Barbara

25. G. Vekinis, M.F. Ashby and F.W.R. Beaumont, Acta Metall. Mater, 38, 1151, (1990) 


\section{LIST OF FIGURES:}

1. The crack element types used in this study to model the main crack and microcracks.

2. The FEM mesh used in the analyses.

3. Variation in the fracture strength for nucleation of two microcrack sizes.

4. Development of the microcrack zone with crack growth. a)- Small microcrack nucleation with higher fracture strength, b)- Large microcrack nucleation with smaller fracture strength.

5. Variation in the shielding/amplification behavior with crack growth for two microcrack sizes.

6. R-curve behavior of the two microcrack sizes.

7. Comparison of the R-curve behavior of continuous nucleation of microcracks with the R-curve behavior of the pre-nucleated the same microcracks. Data is for large microcrack size.

8. Development of the microcrack zones with crack growth. a)- Matrix, b)- Composite with $E_{d} / E_{m}$ of 2.0 and c)-Composite with $E_{\delta} / E_{m}$ of 0.5 .

9. Variation in the shielding/amplification behavior with crack growth in the matrix and in the composites.

10. R-curve behavior of the matrix and the composites.

11. Stress distribution ahead of the main crack tip. a)- without any microcrack formation, b)matrix with microcracks, c)- composite with $E_{d} / E_{m}$ of 2.0 and d)- composite with $E_{d} / E_{m}$ of 0.5 . 


$$
r \cdot S_{y}
$$
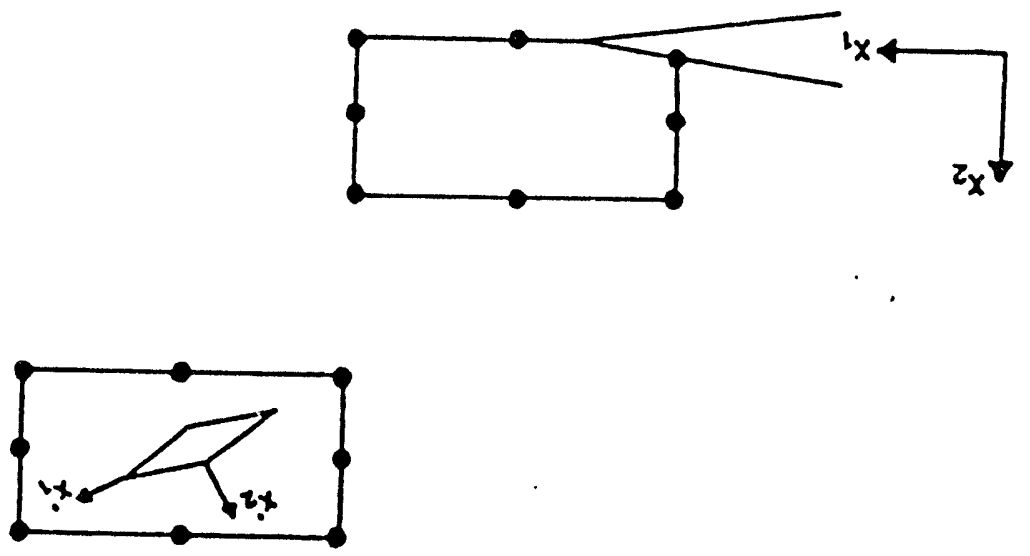

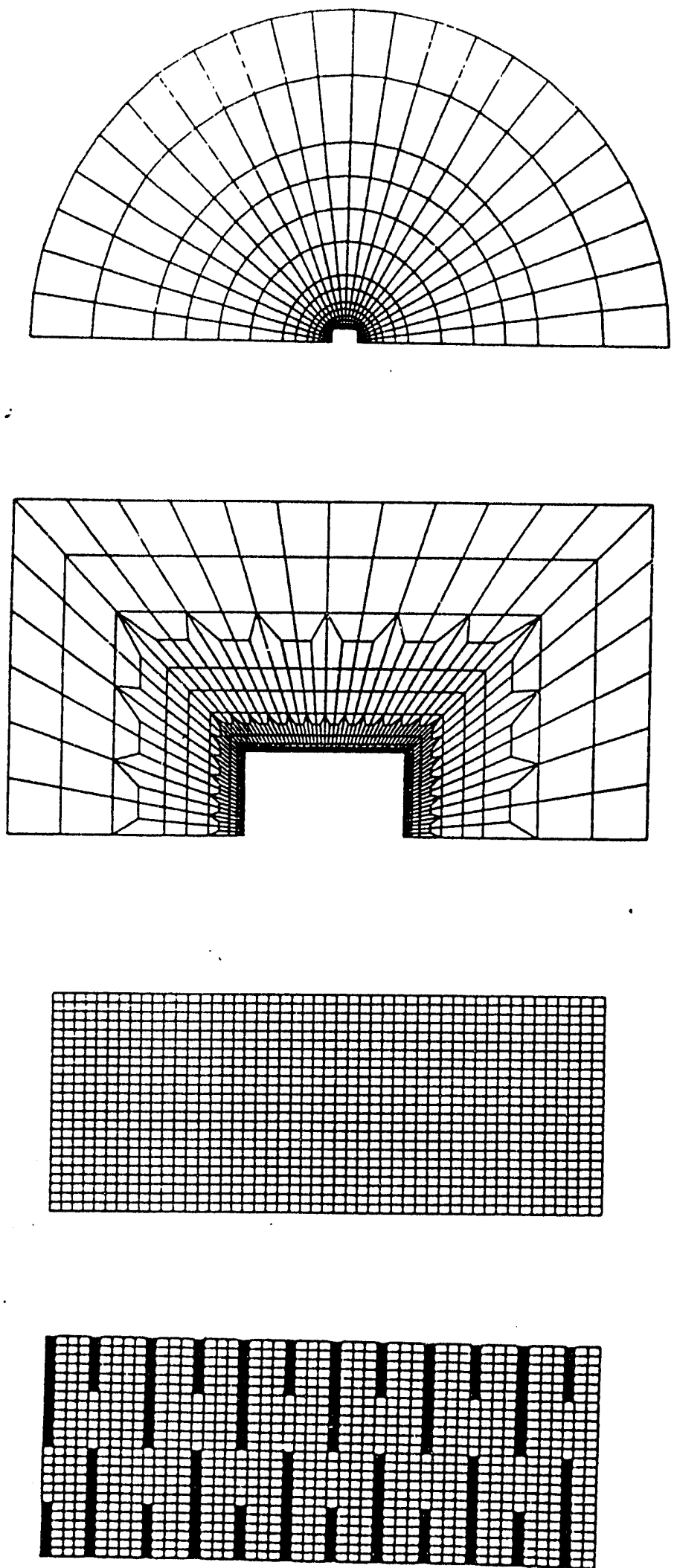

Fig. 2 


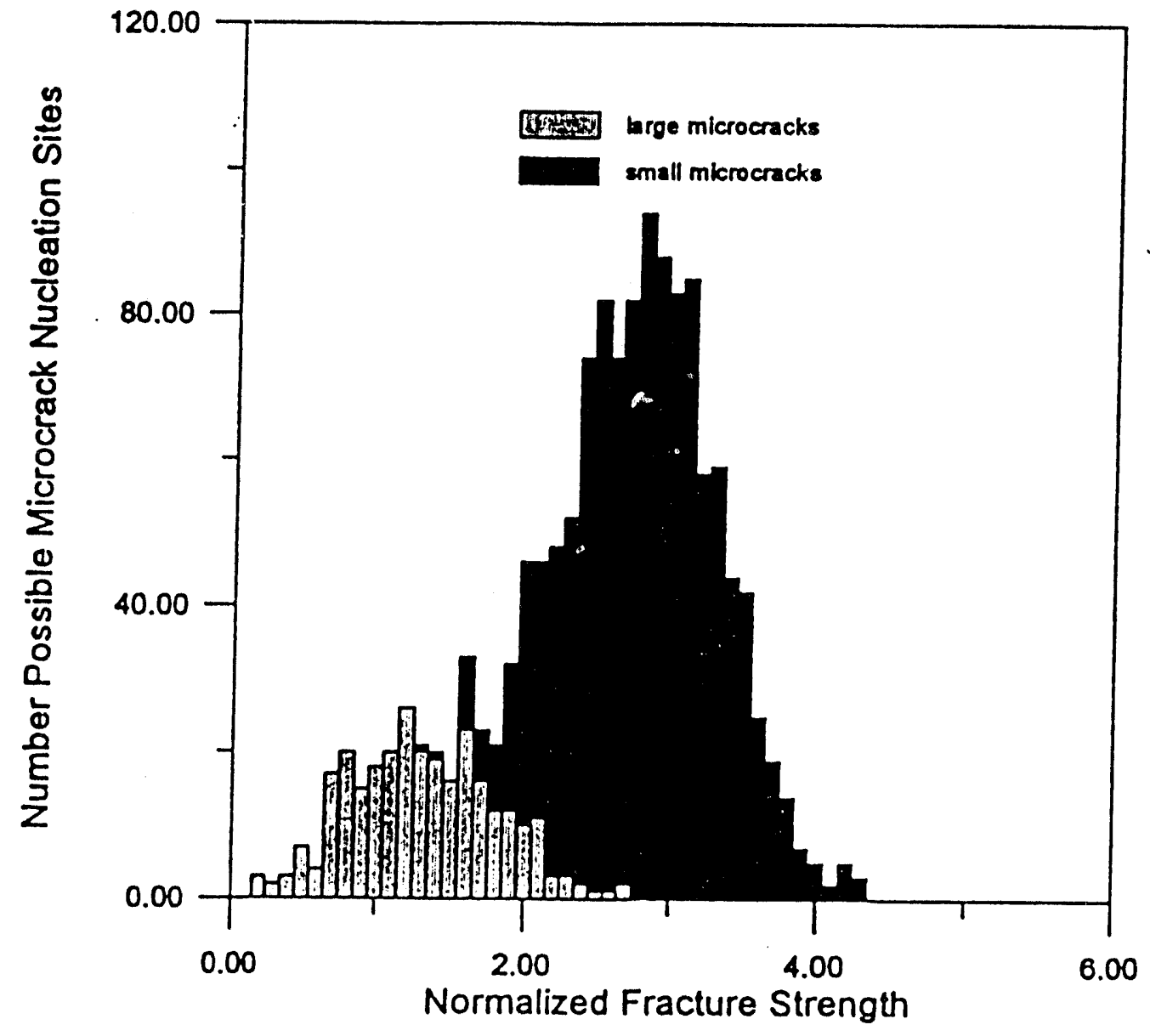

Fip. 
x. $: 1$
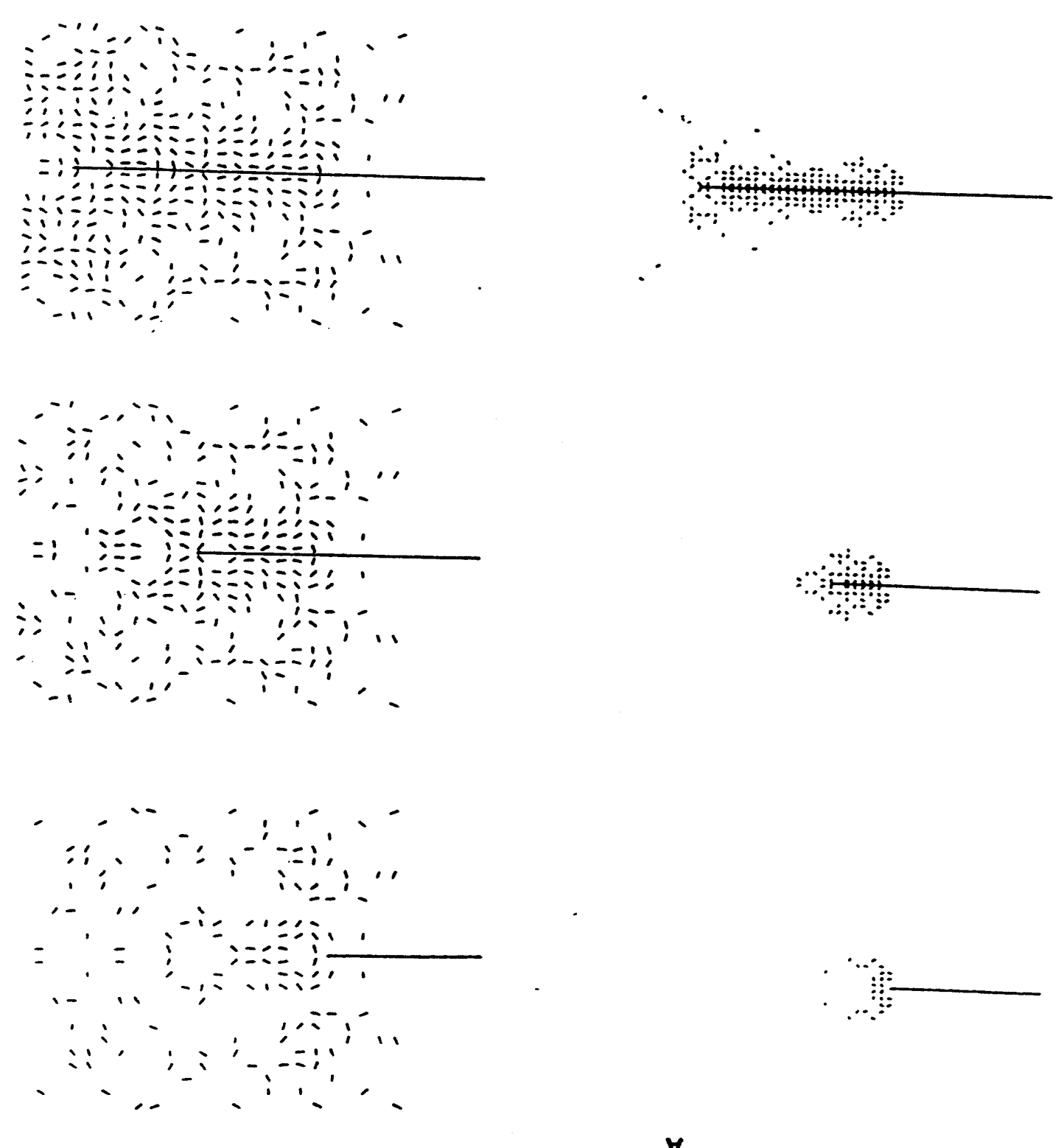

g

$\forall$ 


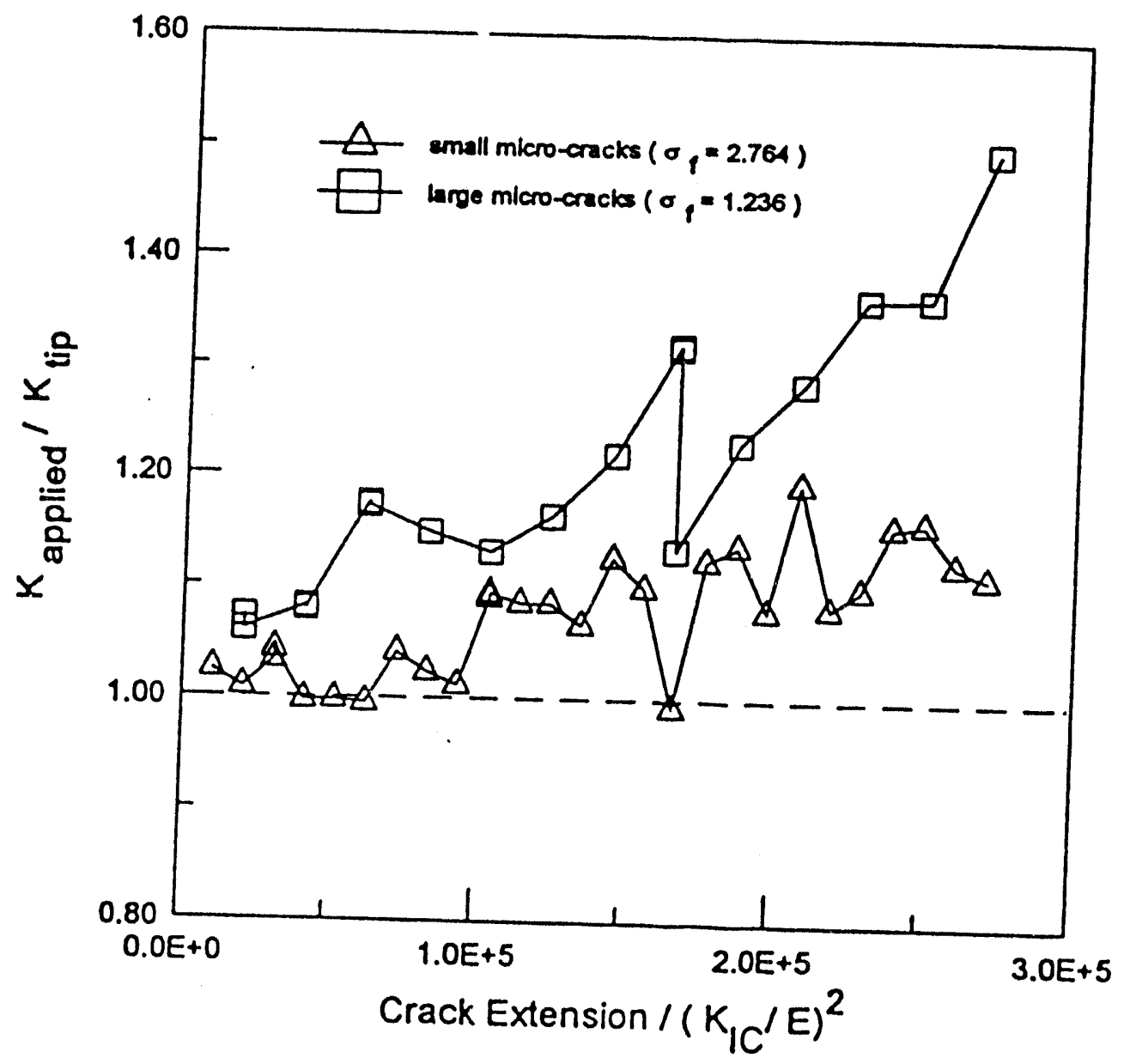

Fig. 5 


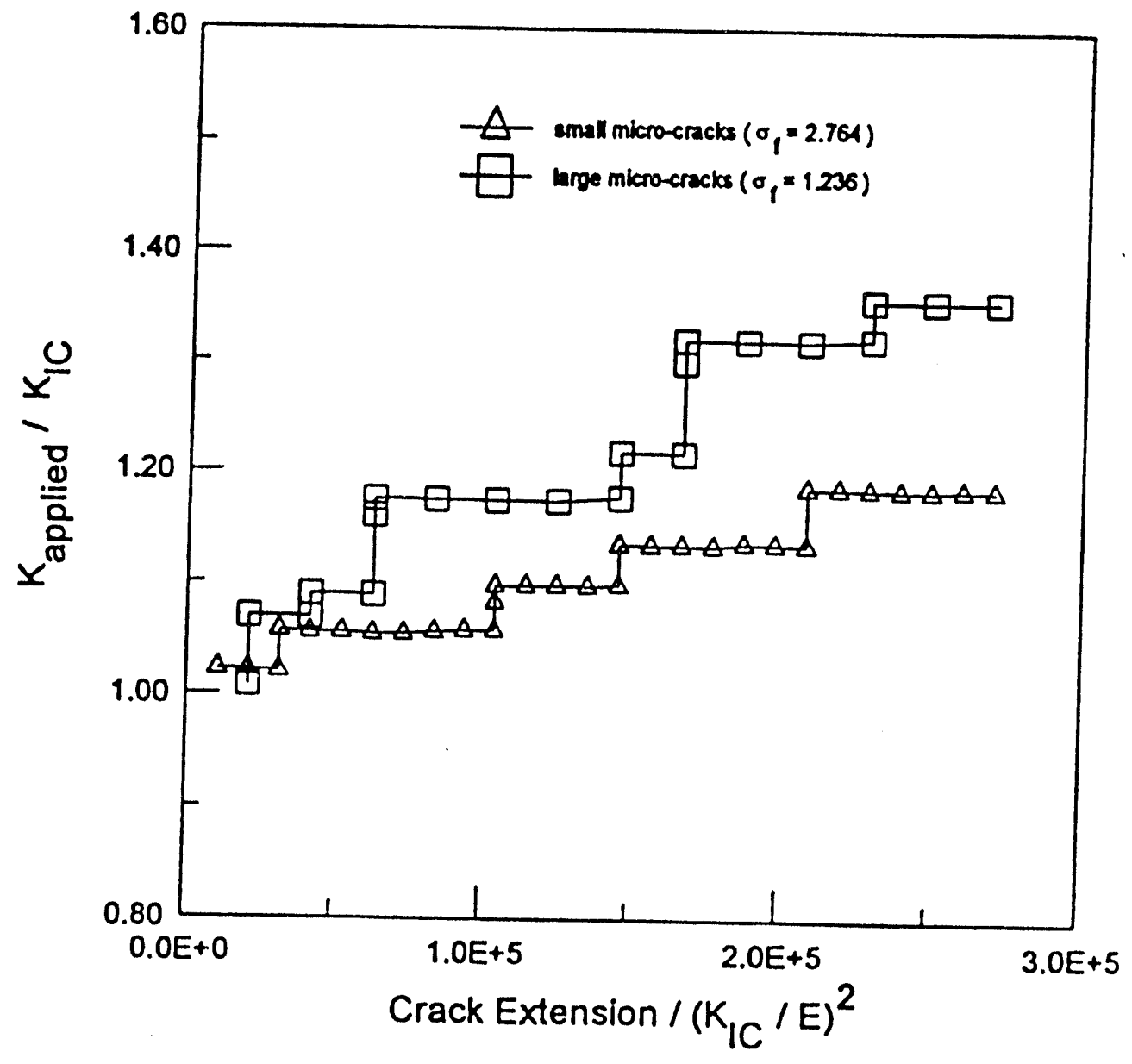

Fip. 6 


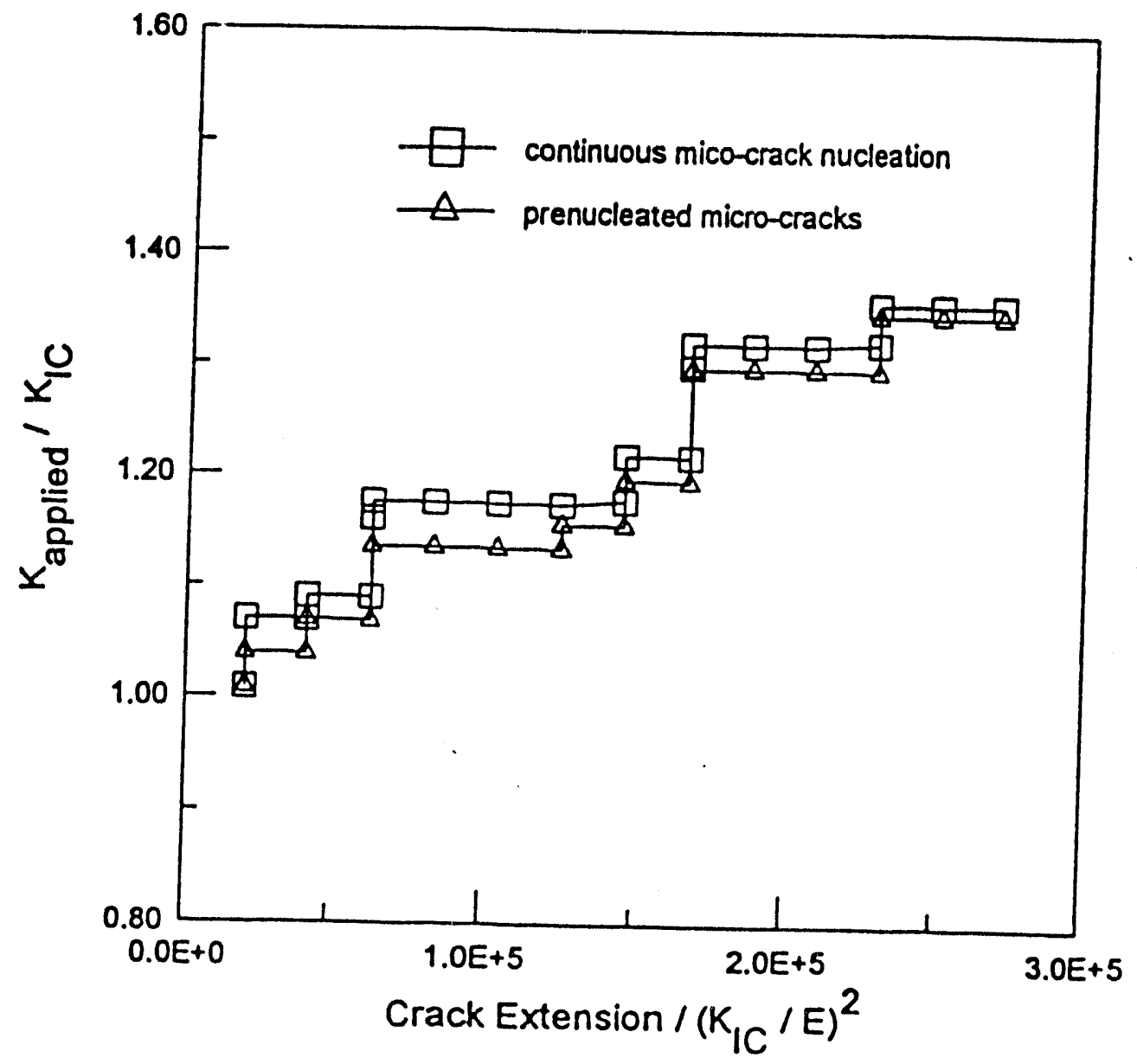

Fig.7 

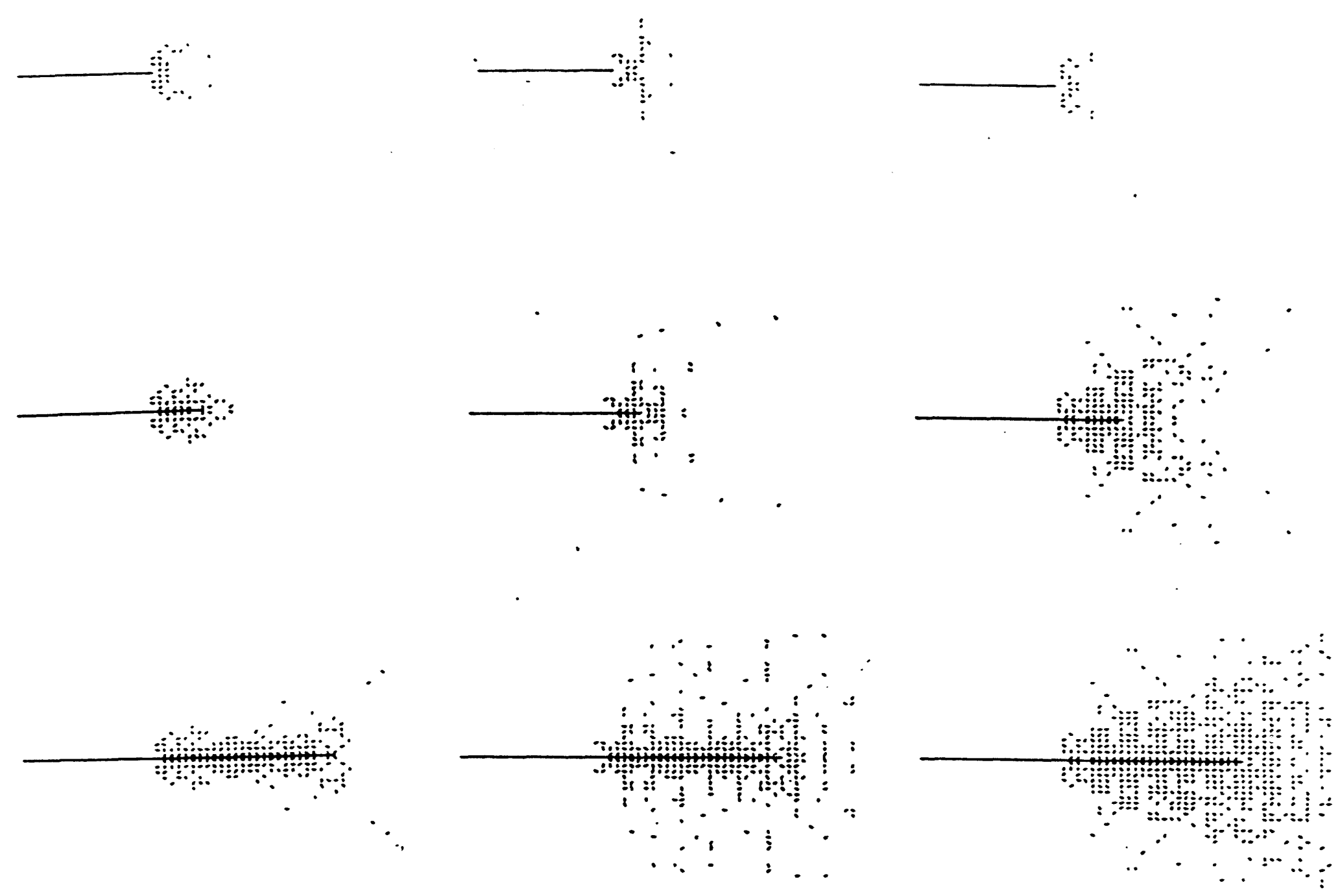

Fij. 6 


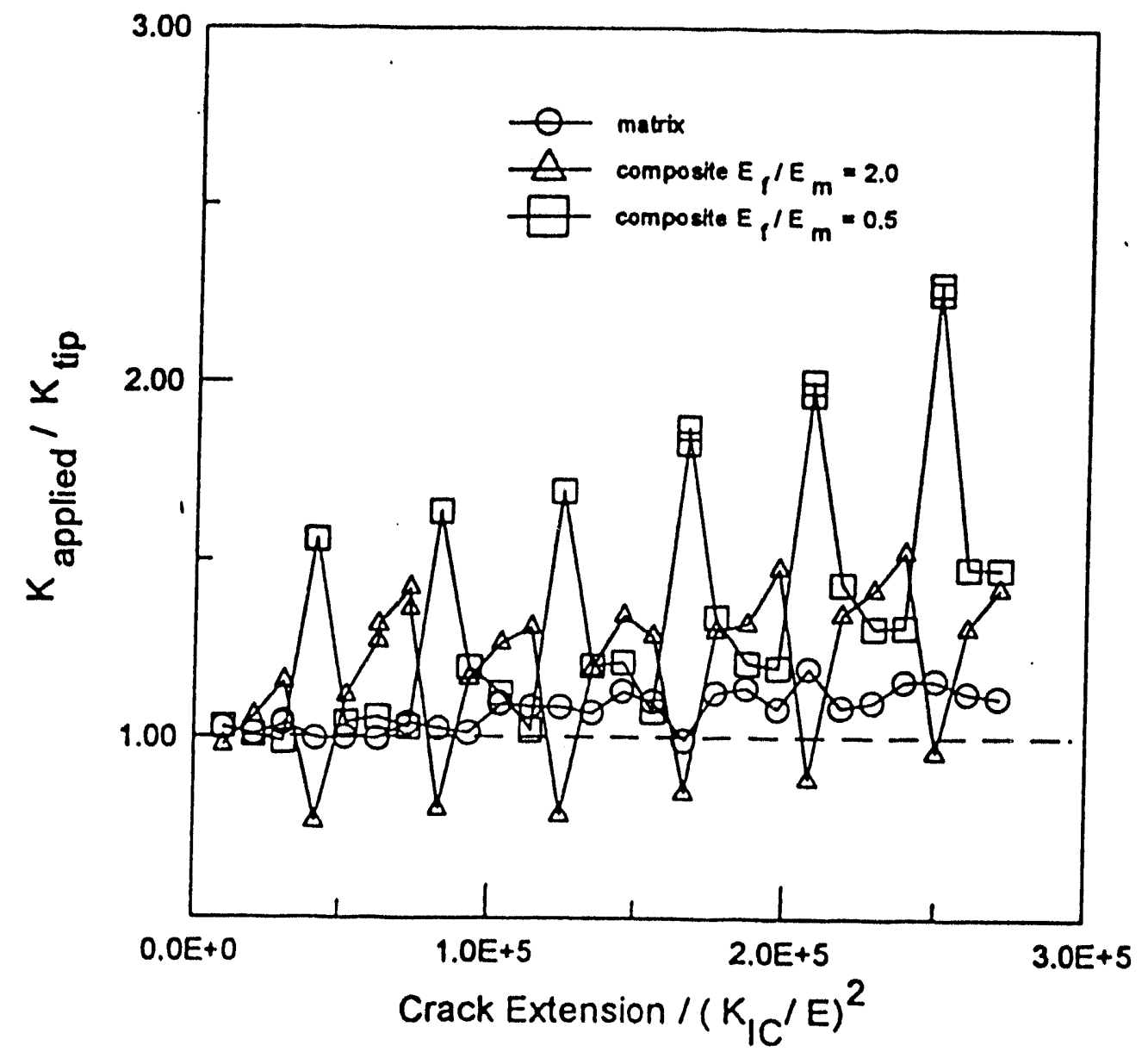

Fij. 9 


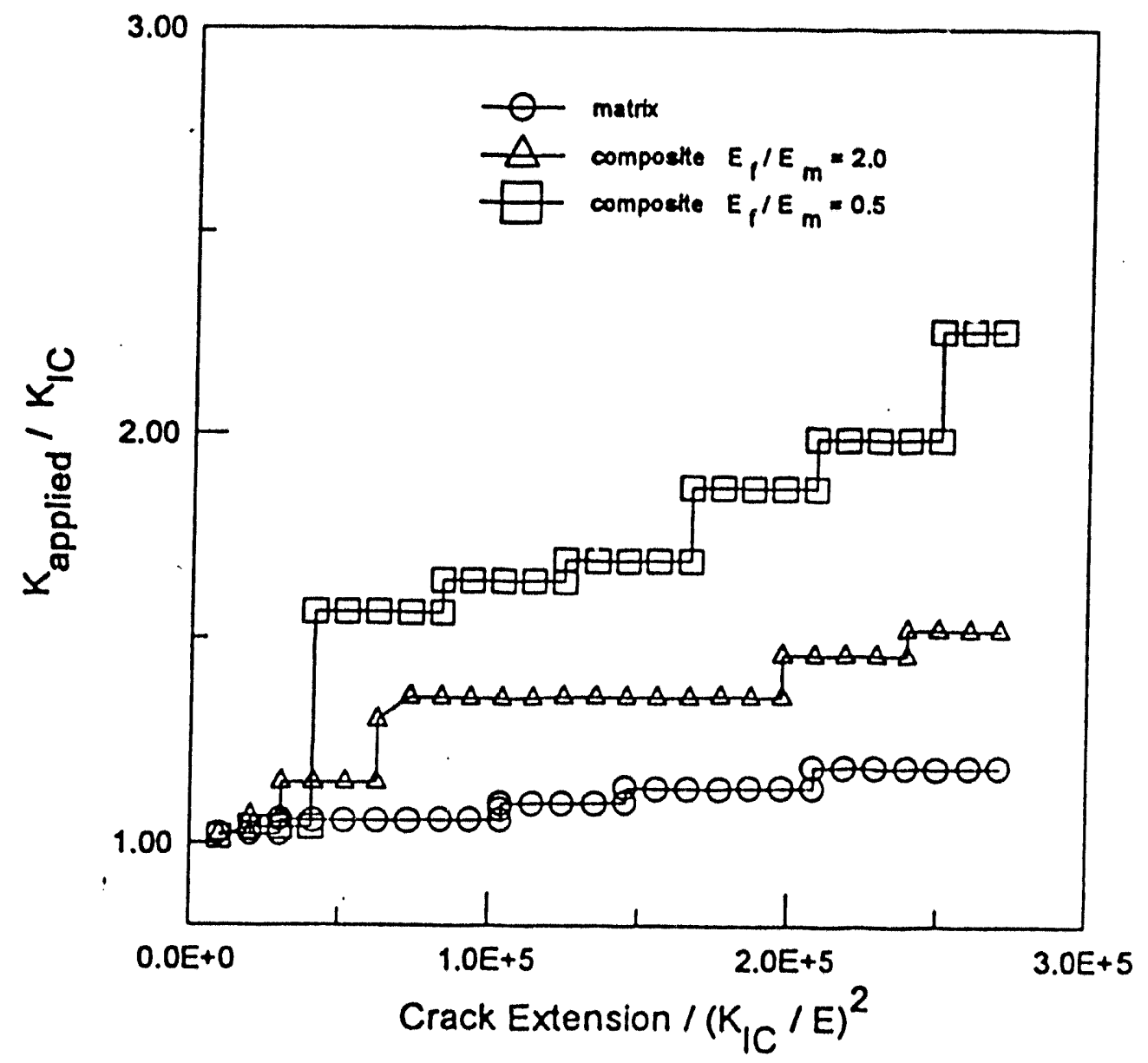

Fig. 10 
$\stackrel{5}{3}$
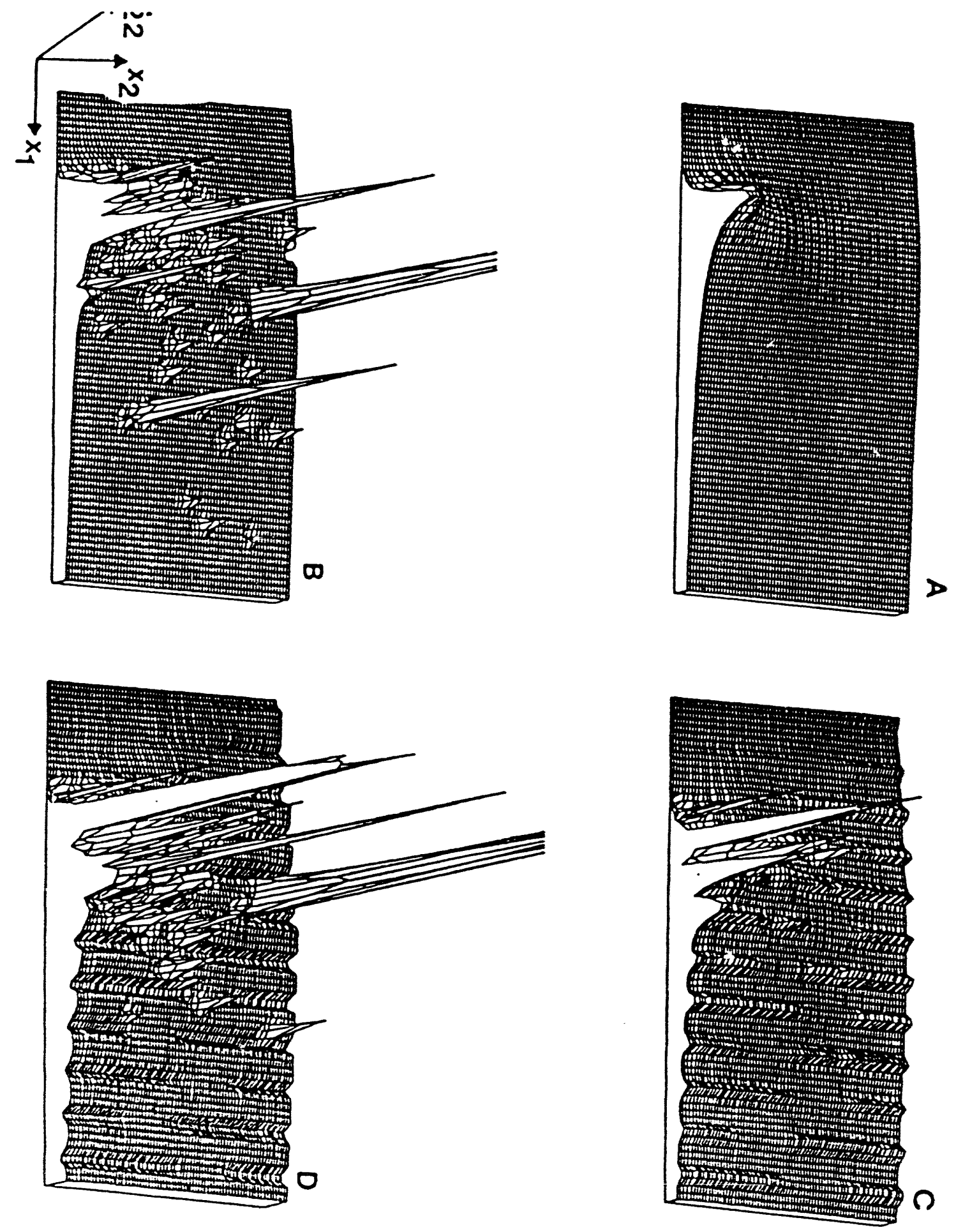


\section{DISCLAMMIXR}

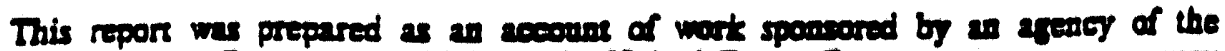

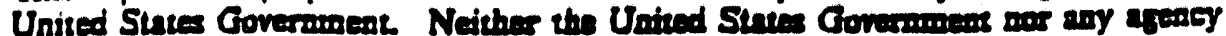

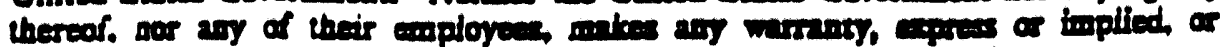

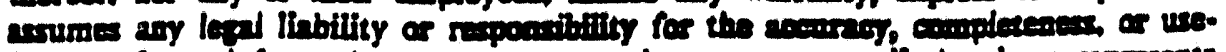

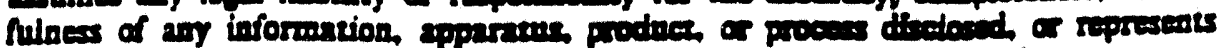

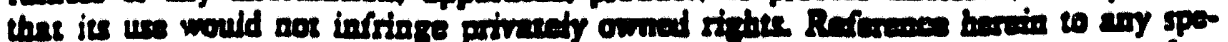

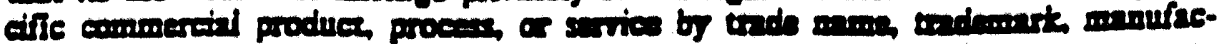

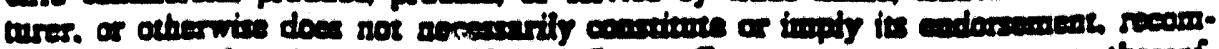

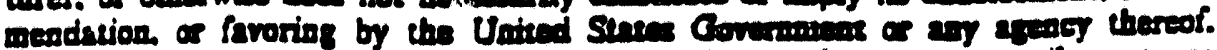

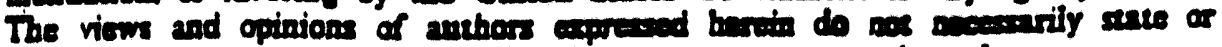

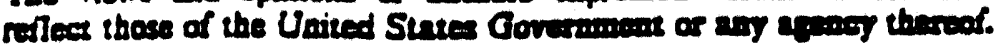



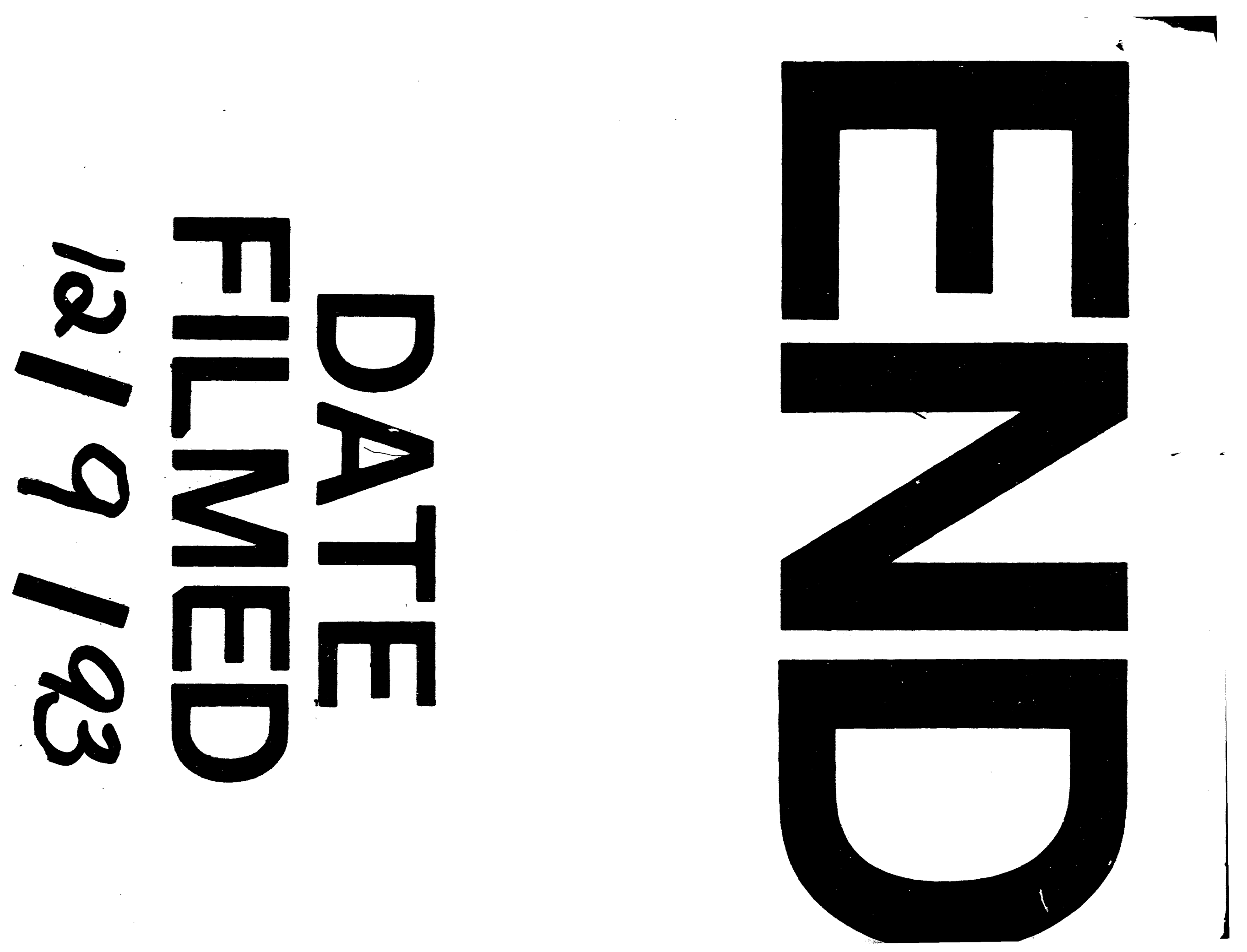


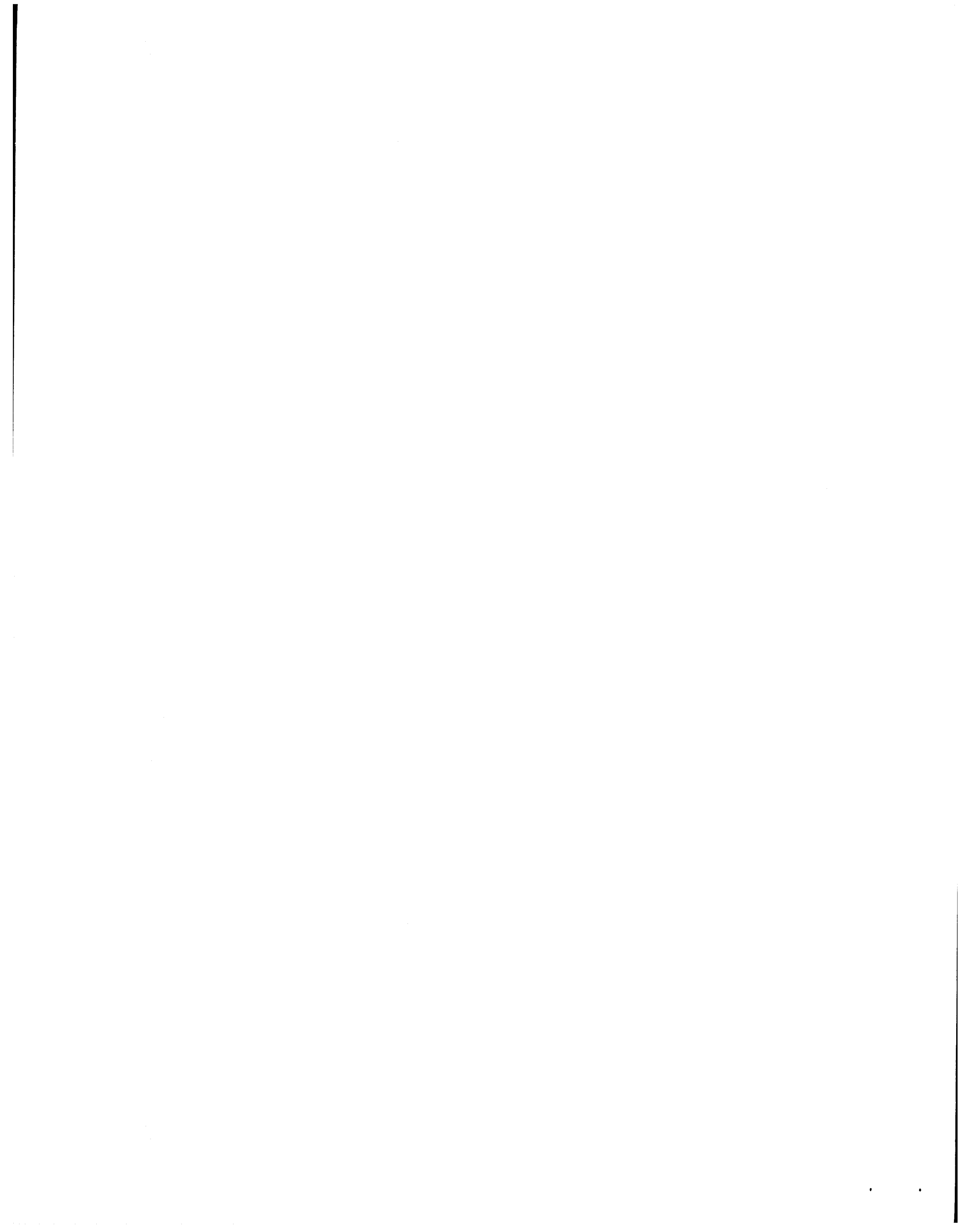

